\title{
Estudo descritivo-comparativo de parâmetros de desempenho aeróbio de atletas profissionais de futebol e futsal
}

\author{
Descriptive-comparative study of aerobic performance \\ parameters between soccer and futsal athletes
}

Bruno Manfredini Baroni ${ }^{1,2}$ William Couto ${ }^{2}$

Ernesto Cesar Pinto Leal Junior ${ }^{1,3}$

\footnotetext{
1 Universidade Federal do Rio Grande do Sul. Escola de Educação Física. Porto Alegre, RS. Brasil.

2 Universidade de Caxias do Sul. Instituto de Medicina do Esporte. Caxias do Sul, RS. Brasil

3 Universidade Nove de Julho. Programa de Pós-Graduação em Ciências da Reabilitação. São Paulo, SP. Brasil
}

Recebido em 30/01/10 Revisado em 19/08/10 Aprovado em 04/01/11
Resumo - Embora futebol e futsal sejam modalidades esportivas com gestos motores semelhantes, as diferenças nas dimensões do local da dinâmica do jogo geram demandas fisiológicas distintas. O objetivo deste estudo foi avaliar e comparar parâmetros representativos da capacidade aeróbia de atletas profissionais de futebol e futsal. Foram realizadas 553 avaliações ergoespirométricas: 367 atletas de futebol (32 goleiros; 335 jogadores de linha) e 186 de futsal (22 goleiros; 164 jogadores de linha). Os resultados foram analisados isoladamente em cada modalidade (comparação entre goleiros e jogadores de linha) e comparados entre futebol e futsal. Goleiros de futebol e futsal possuem menores valores de consumo máximo de oxigênio $\left(\mathrm{VO}_{2 \max }\right)$, segundo limiar ventilatório $\left(\mathrm{LV}_{2}\right)$ e velocidade de obtenção destes limiares em comparação à jogadores de linha das respectivas modalidades. Goleiros de futebol apresentam superioridade nestes parâmetros em relação aos goleiros de futsal. Não foram encontradas diferenças de $\mathrm{VO}_{2 \max }$ e $\mathrm{LV}_{2}$ em jogadores de linha de futebol e futsal, embora os atletas de futebol atinjam os limiares em velocidades mais elevadas. Conclui-se que os goleiros de futebol e futsal apresentam capacidade aeróbica inferior a atletas de linha. Atletas de futebol apresentam melhor desempenho do que atletas de futsal em testes aeróbios.

Palavras-chave: Futebol; Futsal; Consumo de oxigênio; Limiar ventilatório; Desempenho.

Abstract - Although soccer and futsal are sports with similar motor actions, the differences in size and match dynamics result in distinct physiological requirements. The objective of this study was to evaluate and compare parameters of aerobic capacity between professional soccer and futsal athletes. A total of 553 ergospirometric evaluations were performed: 367 soccer players (32 goalkeepers, 335 on-court players) and 186 futsal athletes (22 goalkeepers, 164 on-court players). The results were analyzed separately for each modality (comparison between goalkeepers and on-court players) and compared between soccer and futsal athletes. Soccer and futsal goalkeepers presented lower maximal oxygen uptake $\left(\mathrm{VO}_{2 \max }\right)$, second ventilatory threshold $\left(\mathrm{VT}_{2}\right)$, and speed to reach these thresholds than on-court players of the respective modalities. Soccer goalkeepers were superior in terms of these parameters when compared to futsal goalkeepers. No differences in $\mathrm{VO}_{2 \max }$ or $\mathrm{VT}_{2}$ were observed between soccer and futsal on-court players, although soccer athletes reached the thresholds at higher speeds. In conclusion, soccer and futsal goalkeepers present lower aerobic capacity than on-court players. Soccer players show better performance in aerobic tests than futsal athletes.

Key words: Soccer; Futsal; Oxygen uptake; Ventilatory threshold; Performance. 


\section{INTRODUÇÃO}

$\mathrm{O}$ consumo máximo de oxigênio $\left(\mathrm{VO}_{2 \max }\right)$ pode ser definido como o maior volume de oxigênio por unidade de tempo que um indivíduo consegue captar, transportar e utilizar em nível celular, e é um indicador amplamente utilizado no meio esportivo ou na avaliação funcional de indivíduos não-atletas ${ }^{1}$. Os valores de $\mathrm{VO}_{2 \max }$ podem ser utilizados na prescrição da intensidade do exercício aeróbio e na predição do desempenho físico. No entanto, o estudo dos limiares metabólicos tem sido alvo de inúmeras investigações dentro da fisiologia do exercício, sendo considerado um parâmetro superior ao $\mathrm{VO}_{2 \max }$ na prescrição ${ }^{2}$ e controle do exercício ${ }^{3}$, além da predição da performance atlética ${ }^{4}$, especialmente em esportes de endurance 5 .

Sabe-se que existem dois limiares metabólicos que indicam aumento da produção de lactato. $\mathrm{O}$ primeiro limiar, conhecido como limiar aeróbio, reflete a intensidade do exercício correspondente ao início do acúmulo de lactato sanguíneo, e também pode ser chamado de primeiro limiar ventilatório $\left(\mathrm{LV}_{1}\right)$. O segundo limiar é considerado o ponto de compensação respiratória ${ }^{6}$ ou segundo limiar ventilatório $\left(\mathrm{LV}_{2}\right)$, definido como a intensidade de esforço acima da qual a produção de lactato supera sua própria remoção, provocando hiperventilação, necessária para a diminuição da pressão parcial de dióxido de carbono $\left(\mathrm{PCO}_{2}\right)$ venosa e para o restabelecimento das concentrações de bicarbonato $\left(\mathrm{HCO}^{3-}\right)$, aumentando o $\mathrm{pH}$ plasmático ${ }^{7-8}$. Estas duas variáveis $\left(\mathrm{VO}_{2 \max }\right.$ e $\left.\mathrm{LV}_{2}\right)$ são consideradas parâmetros fundamentais na definição da capacidade aeróbia de um indivíduo ${ }^{9}$ e, embora testes de campo possam ser empregados para estimação de tais variáveis metabólicas, a ergoespirometria consiste em um confiável método não-invasivo capaz de determinar com considerável precisão o $\mathrm{VO}_{2 \max }$ e o $\mathrm{LV}_{2}$ pela quantificação das trocas gasosas realizadas pelo avaliado durante o exercício'.

A capacidade aeróbia dos atletas de futebol tem sido o foco de uma série estudos, de modo que a literatura científica fornece dados relativos a atletas profissionais ${ }^{10-13}$, adolescentes ${ }^{14-16}$, crianÇas $^{17}$ e do sexo feminino ${ }^{18}$. Em relação ao futsal, a lacuna científica que recai sobre a modalidade inclui também a avaliação da capacidade aeróbia. Após detalhada busca nas bases de dados Scielo e PubMed utilizando os termos "futsal" e "indoor soccer" (termo errôneo para designar a modalidade em questão, mas que aparece em alguns estudos que tratam tanto de futsal quanto de futebol society), apenas três estudos ${ }^{19-21}$ foram encontrados acerca da capacidade aeróbia destes atletas.

Por serem duas modalidades esportivas de natureza intermitente e com gestos esportivos semelhantes, mas com distinções relacionadas às dimensões do local de prática e dinâmica do jogo, a comparação entre a capacidade aeróbia de atletas de futebol e futsal já fora enfocada por um estudo prévio ${ }^{20}$. Entretanto, limitações relacionadas à representatividade da amostra incentivaram a realização do presente estudo, que tem como objetivo principal avaliar e comparar parâmetros representativos da capacidade aeróbia de atletas profissionais de futebol e futsal submetidos à avaliação ergoespirométrica.

\section{PROCEDIMENTOS METODOLÓGICOS}

O presente trabalho caracteriza-se como um estudo descritivo com delineamento transversal, no qual foram analisadas 553 avaliações ergoespirométricas de atletas profissionais de futebol e futsal realizadas entre os anos de 2003 e 2007 no Instituto de Medicina do Esporte e Ciências Aplicadas ao Movimento Humano da Universidade de Caxias do Sul (IME-UCS), Caxias do Sul, RS, Brasil.

$\mathrm{Na}$ modalidade futebol, foram consideradas para análise 367 avaliações: 32 goleiros; e 335 jogadores de linha, sendo 50 laterais, 75 zagueiros, 142 meio-campistas e 68 atacantes. Os atletas faziam parte do elenco de três equipes profissionais, participantes, respectivamente, das séries $\mathrm{A}, \mathrm{B}$ e C do Campeonato Brasileiro de Futebol. Na modalidade futsal, foram analisadas 186 avaliações: 22 goleiros; 164 jogadores de linha. Todos eram atletas profissionais vinculados a três equipes participantes da Liga Nacional de Futsal. É importante salientar que as avaliações foram realizadas em diferentes períodos do calendário competitivo das equipes, de acordo com a preferência das comissões técnicas, e que atletas que permaneceram vinculados às equipes participantes, durante os cinco anos de realização do estudo, foram avaliados mais de uma vez.

Como critérios de inclusão, foram adotados os seguintes aspectos: atletas profissionais de futebol ou futsal federados e que fizessem parte do grupo de atletas das equipes participantes do estudo; ausência de cardiopatias, diagnosticadas previamente por eletrocardiograma; ausência de histórico de pneumopatias nos dois meses antecedentes aos testes; ausência de lesões osteomioarticulares que pudessem prejudicar seu desempenho nas avaliações ergoespirométricas; adequada realização 
do teste ergoespirométrico, finalizando o mesmo devido à exaustão promovida pelo exercício e não por motivo outro qualquer; autorização da utilização dos dados pelos autores mediante assinatura de um Termo de Consentimento Livre e Esclarecido. Todas as avaliações de atletas que não obedeceram aos requisitos supracitados foram automaticamente excluídas do estudo.

Os testes ergoespirométricos foram conduzidos por uma mesma equipe composta por profissionais de medicina (com especialização em medicina esportiva), enfermagem e educação física. A execução dos testes ocorreu em um ambiente padronizado, bem iluminado, a uma temperatura controlada de $24^{\circ} \mathrm{C}$. Todos os testes foram realizados em uma mesma esteira ergométrica modelo Super ATL (Inbrasport), sendo utilizado um eletrocardiógrafo digital Elite (Micromed), um analisador de gases modelo $\mathrm{VO}_{2000}$ (MedGraphics) acoplado a um microcomputador equipado com o software específico para tal finalidade (Elite, Micromed), além de clipe nasal esterilizado, eletrodos descartáveis, lâminas de barbear descartáveis, lixas descartáveis e álcool 70\%.

O protocolo adotado consistiu em um teste de esforço progressivo, com inclinação constante de $1^{\circ}$ e aumento gradativo da velocidade da esteira ergométrica. Após um período de 3 minutos de adaptação com caminhada a $4,0 \mathrm{~km} / \mathrm{h}$, o protocolo de avaliação era iniciado com velocidade de 6,0 $\mathrm{km} / \mathrm{h}$. Após o primeiro minuto de teste, a velocidade era incrementada em $2,0 \mathrm{~km} / \mathrm{h}$ (passando então para $8,0 \mathrm{~km} / \mathrm{h}$ ). A partir deste ponto, era realizado um incremento constante de $1,0 \mathrm{~km} / \mathrm{h}$ a cada minuto do teste, sendo que o término do teste era determinado pela exaustão do atleta, que sinalizava ao avaliador a incapacidade em prosseguir. Em seguida, era realizado um período de recuperação a uma velocidade de $4,0 \mathrm{~km} / \mathrm{h}$, mantido pelo tempo necessário até que se atingisse a estabilização da frequência cardíaca e pressão arterial do atleta.

A análise dos dados metabólicos obtidos em cada avaliação foi interpretada por um mesmo médico do esporte com experiência na área. $\mathrm{O} \mathrm{VO}_{2 \max }$ foi considerado o valor pico de consumo de oxigênio mensurado pelo analisador de gases durante a avaliação. $\mathrm{O} \mathrm{LV}_{2}$ foi identificado mediante o uso do equivalente ventilatório de dióxido de carbono $\left(\mathrm{VE} / \mathrm{VCO}_{2}\right)$, em análise realizada perante plotagem dos dados metabólicos em gráfico, considerando o limiar anaeróbio do indivíduo o aumento abrupto do $\mathrm{VE} / \mathrm{VCO}_{2}$, de acordo com os critérios propostos por McLellan 22 .
As variáveis adotadas para análise foram: idade; massa corporal; estatura; $\mathrm{VO}_{2 \max } ;$ velocidade em que foi atingido o $\mathrm{VO}_{2 \max } ; \mathrm{LV}_{2}$; velocidade em que foi atingido o $\mathrm{LV}_{2}$; e percentual do $\mathrm{VO}_{2 \max }$ em que foi atingido o $\mathrm{LV}_{2}$. Primeiramente, foi realizada análise estatística dentro de cada modalidade, comparando os valores obtidos pelos goleiros e pelos jogadores de linha de futebol e futsal, respectivamente. Em seguida, foram realizadas comparações entre os goleiros de futebol e goleiros de futsal, bem como entre os jogadores de linha de futebol e os jogadores de linha de futsal. Todas as variáveis, após verificação da homogeneidade amostral pelo teste de Levene, foram comparadas entre as posições ou modalidades por meio de um teste $t$-Student para amostras independentes. $\mathrm{O}$ procedimento estatístico foi realizado no software SPSS 16.0 e foi adotado um nível de significância de 5\% ( $<<0,05)$.

\section{RESULTADOS}

A Tabela 1 apresenta características antropométricas e de desempenho no protocolo de avaliação ergoespirométrica de atletas que atuam como goleiros e jogadores de linha nas respectivas modalidades futebol e futsal. Fica evidenciado que os goleiros de ambas as modalidades esportivas apresentam estatura e massa corporal significativamente maior que os jogadores de linha de futebol e futsal, respectivamente. Além disso, destaca-se que os valores de $\mathrm{VO}_{2 \max }, \mathrm{LV}_{2}$ e velocidade de obtenção de $\mathrm{VO}_{2 \max }$ e $\mathrm{LV}_{2}$ dos goleiros são inferiores aos dos jogadores de linha, tanto no futebol quanto no futsal. Entretanto, em ambas as modalidades os goleiros e os jogadores de linha atingiram o $\mathrm{LV}_{2}$ em um mesmo percentual do $\mathrm{VO}_{2 \max }$.

As diferenças observadas entre atletas que atuam como goleiros e jogadores de linha nas duas modalidades incentivaram a separação das posições táticas para a realização da comparação entre atletas de futebol e futsal, conforme apresentado na Tabela 2. Os resultados demonstram que os goleiros de futebol são mais altos que os de futsal, além de apresentar valores superiores de $\mathrm{VO}_{2 \max }, \mathrm{LV}_{2} \mathrm{e}$ velocidade de obtenção de $\mathrm{VO}_{2 \max }$ e $\mathrm{LV}_{2}$. Entre os jogadores de linha, também se observa que os atletas de futebol são mais altos. Além disso, embora jogadores de linha de futebol e futsal apresentem valores similares de $\mathrm{VO}_{2 \max }, \mathrm{LV}_{2}$ e percentual do $\mathrm{VO}_{2 \max }$ em que é atingido o $\mathrm{LV}_{2}$, os atletas de futebol atingem estes limiares em maiores velocidades que os atletas de futsal 
Tabela 1. Comparação de características físicas e de desempenho na avaliação ergoespirométrica entre goleiros e jogadores de linha de futebol e futsal.

\begin{tabular}{|c|c|c|c|}
\hline & Goleiros & Jogadores de linha & Valor de "p" \\
\hline \multicolumn{4}{|l|}{ Futebol } \\
\hline Amostra & $N=32$ & $N=335$ & \\
\hline Idade (anos) & $24,97 \pm 5,35$ & $23,98 \pm 4,42$ & 0,234 \\
\hline Massa corporal (kg) & $82,87 \pm 5,94$ & $74,96 \pm 6,62$ & 0,000 \\
\hline Estatura $(\mathrm{m})$ & $1,87 \pm 0,04$ & $1,78 \pm 0,06$ & 0,000 \\
\hline $\mathrm{VO}_{2 \max }(\mathrm{mL} / \mathrm{kg} \cdot \min )$ & $56,20 \pm 4,76$ & $59,43 \pm 4,81$ & 0,000 \\
\hline Velocidade $\mathrm{VO}_{2 \max }(\mathrm{km} / \mathrm{h})$ & $17,03 \pm 0,93$ & $17,61 \pm 0,93$ & 0,001 \\
\hline $\mathrm{LV}_{2}(\mathrm{~mL} / \mathrm{kg} \cdot \min )$ & $49,80 \pm 4,20$ & $52,21 \pm 4,67$ & 0,005 \\
\hline Velocidade $\mathrm{LV}_{2}(\mathrm{~km} / \mathrm{h})$ & $14,47 \pm 1,05$ & $14,88 \pm 0,93$ & 0,018 \\
\hline $\mathrm{LV}_{2}\left(\% \mathrm{VO}_{2 \max }\right)$ & $88,68 \pm 3,86$ & $87,89 \pm 4,16$ & 0,302 \\
\hline \multicolumn{4}{|l|}{ Futsal } \\
\hline Amostra & $N=22$ & $N=164$ & \\
\hline Idade (anos) & $22,77 \pm 5,25$ & $23,92 \pm 5,40$ & 0,349 \\
\hline Massa corporal (kg) & $85,95 \pm 10,23$ & $74,49 \pm 8,12$ & 0,000 \\
\hline Estatura $(\mathrm{m})$ & $1,80 \pm 0,05$ & $1,76 \pm 0,06$ & 0,006 \\
\hline $\mathrm{VO}_{2 \max }(\mathrm{mL} / \mathrm{kg} \cdot \min )$ & $50,66 \pm 5,24$ & $58,99 \pm 5,86$ & 0,000 \\
\hline Velocidade $\mathrm{VO}_{2 \max }(\mathrm{km} / \mathrm{h})$ & $15,64 \pm 1,00$ & $17,12 \pm 1,02$ & 0,000 \\
\hline $\mathrm{LV}_{2}(\mathrm{~mL} / \mathrm{kg} \cdot \mathrm{min})$ & $45,62 \pm 4,71$ & $52,01 \pm 5,57$ & 0,000 \\
\hline Velocidade $\mathrm{LV}_{2}(\mathrm{~km} / \mathrm{h})$ & $13,32 \pm 1,04$ & $14,60 \pm 0,88$ & 0,000 \\
\hline $\mathrm{LV}_{2}\left(\% \mathrm{VO}_{2 \max }\right)$ & $90,23 \pm 6,27$ & $88,29 \pm 5,76$ & 0,143 \\
\hline
\end{tabular}

Tabela 2. Comparação de características físicas e de desempenho na avaliação ergoespirométrica entre atletas de futebol e futsal que atuam como goleiros e jogadores de linha.

\begin{tabular}{|c|c|c|c|}
\hline & Futebol & Futsal & Valor de "p" \\
\hline \multicolumn{4}{|l|}{ Goleiros } \\
\hline Amostra & $N=32$ & $N=22$ & \\
\hline Idade (anos) & $24,97 \pm 5,35$ & $22,77 \pm 5,25$ & 0,142 \\
\hline Massa corporal (kg) & $82,87 \pm 5,94$ & $85,95 \pm 10,23$ & 0,213 \\
\hline Estatura (m) & $1,87 \pm 4,38$ & $1,80 \pm 5,47$ & 0,000 \\
\hline $\mathrm{VO}_{2 \max }(\mathrm{mL} / \mathrm{kg} \cdot \min )$ & $56,20 \pm 4,76$ & $50,66 \pm 5,24$ & 0,000 \\
\hline Velocidade $\mathrm{VO}_{2 \max }(\mathrm{km} / \mathrm{h})$ & $17,03 \pm 0,93$ & $15,64 \pm 1,00$ & 0,000 \\
\hline $\mathrm{LV}_{2}(\mathrm{~mL} / \mathrm{kg} \cdot \mathrm{min})$ & $49,80 \pm 4,20$ & $45,62 \pm 4,71$ & 0,001 \\
\hline Velocidade $\mathrm{LV}_{2}(\mathrm{~km} / \mathrm{h})$ & $14,47 \pm 1,05$ & $13,32 \pm 1,04$ & 0,000 \\
\hline $\mathrm{LV}_{2}\left(\% \mathrm{VO}_{2 \max }\right)$ & $88,68 \pm 3,86$ & $90,23 \pm 6,27$ & 0,309 \\
\hline \multicolumn{4}{|l|}{ Jogadores de linha } \\
\hline Amostra & $N=335$ & $N=164$ & \\
\hline Idade (anos) & $23,98 \pm 4,42$ & $23,92 \pm 5,40$ & 0,903 \\
\hline Massa corporal (kg) & $74,96 \pm 6,62$ & $74,49 \pm 8,12$ & 0,520 \\
\hline Estatura (m) & $1,78 \pm 0,06$ & $1,76 \pm 0,06$ & 0,000 \\
\hline $\mathrm{VO}_{2 \max }(\mathrm{mL} / \mathrm{kg} \cdot \min )$ & $59,43 \pm 4,81$ & $58,99 \pm 5,86$ & 0,412 \\
\hline Velocidade $\mathrm{VO}_{2 \max }(\mathrm{km} / \mathrm{h})$ & $17,62 \pm 0,93$ & $17,12 \pm 1,02$ & 0,000 \\
\hline $\mathrm{LV}_{2}(\mathrm{~mL} / \mathrm{kg} \cdot \mathrm{min})$ & $52,21 \pm 4,67$ & $52,01 \pm 0,93$ & 0,677 \\
\hline Velocidade $\mathrm{LV}_{2}(\mathrm{~km} / \mathrm{h})$ & $14,88 \pm 0,92$ & $14,60 \pm 0,88$ & 0,002 \\
\hline $\mathrm{LV}_{2}\left(\% \mathrm{VO}_{2 \max }\right)$ & $87,89 \pm 4,16$ & $88,29 \pm 5,76$ & 0,381 \\
\hline
\end{tabular}

\section{DISCUSSÃO}

Os valores de $\mathrm{VO}_{2 \max }$ encontrados no presente estudo para os atletas de futebol condizem com valores reportados na literatura para atletas pro- fissionais desta modalidade ${ }^{11-13,23}$. Entretanto, os valores relativos ao $\mathrm{LV}_{2}$ mostram-se superiores aos relatados ${ }^{11}$, porém ocorrendo em similares percentuais do $\mathrm{VO}_{2 \max }{ }^{10,23}$. Stolen et al. ${ }^{23}$ realizaram uma abrangente revisão sobre a fisiologia do futebol 
na qual, após observação de mais de 30 estudos na área envolvendo atletas de diferentes níveis competitivos, concluíram que o $\mathrm{VO}_{2 \max }$ em atletas de futebol do sexo masculino varia entre 50 e 75 $\mathrm{mL} / \mathrm{kg}$.min. Díaz et al. ${ }^{12}$ demonstraram a evolução da preparação física no futebol, apresentando resultados de 248 atletas profissionais de elite, nas décadas de 70, 80 e 90, onde os valores do $\mathrm{VO}_{2 \max }$ foram respectivamente de 52,54 e $57 \mathrm{~mL} / \mathrm{kg}$.min. Arnasson et al. ${ }^{13}$, na divisão de elite da Islândia, encontraram valores de $\mathrm{VO}_{2 \max }$ de $63,2 \mathrm{~mL} / \mathrm{kg}$.min Al-Hazzaa et al. ${ }^{11}$, avaliando 23 atletas da seleção saudita de futebol, encontraram valores médios de $56,8 \mathrm{~mL} / \mathrm{kg} \cdot \mathrm{min}$ e $43,6 \mathrm{~mL} / \mathrm{kg} \cdot \min$ para $\mathrm{VO}_{2 \max }$ e $\mathrm{LV}_{2}$, respectivamente. Também em relação ao $\mathrm{LV}_{2}$, já fora observado que este estágio é atingido entre $76,6 \%$ e $90,3 \%$ do $\mathrm{VO}_{2 \max }{ }^{10,23}$.

Em relação aos atletas de futsal, os valores obtidos no presente estudo para $\mathrm{VO}_{2 \max }$ e $\mathrm{LV}_{2}$, de maneira geral, se aproximam dos valores encontrados pelos trabalhos mencionados ${ }^{19-21}$. Entretanto, o presente estudo observou o $\mathrm{LV}_{2}$ dos atletas de futsal ocorrendo em percentagens do $\mathrm{VO}_{2 \max }$ mais elevadas que as até então reportadas ${ }^{21}$. Lima et al. ${ }^{19}$, avaliando 13 atletas da modalidade, encontram um $\mathrm{VO}_{2 \max }$ de $62,8 \mathrm{~mL} / \mathrm{kg}$.min em medida direta (ergoespirometria) e $58,5 \mathrm{~mL} / \mathrm{kg} \cdot \mathrm{min}$ em medida indireta (teste de campo de 3200 metros). No estudo de Leal Junior et al..$^{20}$, após a avaliação de 12 atletas profissionais de futsal por meio de ergoespirometria, os autores encontraram um $\mathrm{VO}_{2 \max }$ de $55,7 \mathrm{~mL} /$ kg.min. Já Castagna et al. ${ }^{21}$, avaliando oito atletas profissionais, observaram $\mathrm{VO}_{2 \max }$ variando entre 53,8 e $75,8 \mathrm{~mL} / \mathrm{kg} \cdot \min$ (média de $64,8 \mathrm{~mL} / \mathrm{kg} \cdot \mathrm{min}$ ) e um $\mathrm{LV}_{2}$ entre 36,0 e $56,0 \mathrm{~mL} / \mathrm{kg}$.min (média de $46 \mathrm{~mL} / \mathrm{kg} . \mathrm{min}$ ), correspondendo a 65-77\% (média de $71,0 \%)$ do $\mathrm{VO}_{2 \max }$.

A diferença de capacidade aeróbia entre goleiros e jogadores de linha no futebol já foi demonstrada previamente ${ }^{13}$. No entanto, apesar de esperada em função das demandas do jogo, a diferença entre goleiros e jogadores de linha no futsal parece não ter sido reportada até então. Assim, a confirmação das distintas capacidades aeróbias entre goleiros e jogadores de linha das duas modalidades incentivou a análise separada por posições táticas de jogo, comparando goleiros de futebol com goleiros de futsal e jogadores de linha de futebol com jogadores de linha de futsal para a obtenção do principal objetivo do presente estudo, que se foca na comparação entre estas duas modalidades que apresentam gestos esportivos similares, mas demandas fisiológicas bem particulares. Durante um jogo de futebol, atletas de elite apresentam um consumo médio de aproximadamente $70 \%$ do $\mathrm{VO}_{2 \max }{ }^{24-26}$. Em relação à demanda metabólica no futsal, um único estudo ${ }^{21}$ verificou o $\mathrm{VO}_{2}$ durante um jogo simulado, situação na qual os atletas atuaram em uma média de $76 \%$ do $\mathrm{VO}_{2 \max }$, chegando a atingir picos de até $99 \%$ do $\mathrm{VO}_{2 \max }$ mensurado em laboratório. Entretanto, acredita-se que tais mensurações possam ser subestimadas, tendo em vista que o equipamento acoplado ao atleta pode inibir o seu desempenho ${ }^{23}$.

Os resultados do presente estudo demonstram haver superioridade na capacidade aeróbia dos goleiros de futebol em relação aos goleiros de futsal. Esta superioridade é representada pelos maiores valores encontrados nos parâmetros fisiológicos avaliados $\left(\mathrm{VO}_{2 \max }\right.$ e $\left.\mathrm{LV}_{2}\right)$ e traduzida em desempenho através da maior velocidade em que os goleiros de futebol atingiram estes parâmetros. Entre os atletas de linha, não foram verificadas diferenças significativas entre os valores de $\mathrm{VO}_{2 \max } \mathrm{e} \mathrm{LV}_{2}$. No entanto, a superioridade dos atletas de futebol em relação à velocidade de obtenção destes limiares sugere uma melhor condição física destes atletas, uma vez que os atletas de futebol apresentaram uma maior tolerância ao exercício incremental a que foram submetidos e, consequentemente, suportaram o exercício por períodos de tempo mais longos que os atletas de futsal antes de atingir a exaustão.

Os dados do presente estudo concordam, em parte, com os resultados apresentados por Leal Junior et al. $^{20}$, que observaram similaridade no $\mathrm{VO}_{2 \max }$ entre atletas das duas modalidades em questão. Entretanto, enquanto estes autores encontraram um $\mathrm{LV}_{2}$ sendo atingido precocemente pelos atletas de futsal, os resultados do presente estudo não encontram diferenças entre os atletas de linha das duas modalidades. Talvez, a análise em conjunto de goleiros e jogadores de linha de futebol e futsal possa ter influenciado os resultados encontrados por Leal Junior et al..$^{20}$, o que levou os autores a sugerir que o futsal seria um esporte de predominância anaeróbia em relação ao futebol, de modo que os atletas estariam mais bem adaptados ao exercício neste nível de intensidade e, por isso, teriam apresentado uma capacidade superior de manutenção no exercício após atingir o $\mathrm{LV}_{2}$, uma hipótese que não pode ser suportada pelos resultados do presente estudo.

Considerando-se somente a frequência cardíaca média observada em atletas profissionais durante jogos de futebol (85-93\% da $\left.\mathrm{FC}_{\max }\right)^{23} \mathrm{e}$ futsal $\left(90 \% \text { da } \mathrm{FC}_{\max }\right)^{21,27}$, poder-se-ia inferir que a 
intensidade média de exercício imposta pelas duas modalidades é semelhante. Entretanto, deve-se levar em conta que os atletas de futebol percorrem, aproximadamente, $10-12 \mathrm{~km}^{23}$ durante dois tempos de 45 minutos de jogo, enquanto os atletas de futsal percorrem algo em torno de $4 \mathrm{~km}^{27}$, durante dois tempos de 20 minutos, com cronômetro parado nas interrupções do jogo (saídas de bola, faltas, etc.). Ainda deve ser levado em consideração o fato de a regra do futsal permitir número ilimitado de substituição de jogadores, fazendo com que os mesmos permanecem em quadra por períodos bastante curtos quando comparados aos atletas de futebol que, em sua maioria, permanecem em campo os 90 minutos. Assim, os resultados obtidos no presente estudo podem ser explicados pela adaptação dos atletas às demandas dos seus respectivos esportes. Como já fora demonstrado ${ }^{24-25}$, o $\mathrm{VO}_{2 \max }$ está diretamente relacionado com a distância percorrida em uma partida, o que justificaria a melhor capacidade de resistência dos atletas de futebol a maiores velocidades e tempos de permanência no exercício antes da exaustão.

Uma limitação a ser apontada no presente estudo diz respeito ao fato de as avaliações ergoespirométricas terem sido realizadas em diferentes períodos da temporada, de acordo com o interesse das equipes. Assim, embora as avaliações laboratoriais tenham sido padronizadas em questões relativas ao protocolo e condições ambientais (luminosidade, temperatura), avaliaram-se atletas em diferentes estágios de treinamento de acordo com a periodização implementada por cada comissão técnica. Entretanto, ressalta-se a importância deste trabalho por tratar-se de uma amostra numericamente expressiva, bem superior a maioria dos trabalhos da área, além de ser composta por atletas que representam os mais altos níveis competitivos do futebol e futsal nacional.

\section{CONCLUSÕES}

Os resultados obtidos nas 553 avaliações de atletas profissionais de futebol e futsal utilizadas na análise do presente estudo permitem concluir que: (1) os goleiros de futebol e futsal possuem capacidade aeróbia inferior aos jogadores de linha das respectivas modalidades, observada pelos menores valores de $\mathrm{VO}_{2 \max }, \mathrm{LV}_{2}$ e velocidade de obtenção de $\mathrm{VO}_{2 \max } \mathrm{e}$ $\mathrm{LV}_{2}$; (2) os goleiros de futebol apresentam maiores valores de $\mathrm{VO}_{2 \max }$, $\mathrm{LV} 2$ e velocidade de obtenção de $\mathrm{VO}_{2 \max }$ e $\mathrm{LV}_{2}$ do que os goleiros de futsal; e (3) não houve diferença nos valores de $\mathrm{VO}_{2 \max }$ e $\mathrm{LV}_{2}$ de jogadores de linha de linha de futebol e futsal, no entanto, os atletas de futebol atingem os respectivos limiares em velocidades superiores aos atletas de futsal (ou seja, permanecem mais tempo em exercício antes da exaustão), sugerindo que os atletas de futebol possuem um melhor preparo físico para este tipo de avaliação, possivelmente atribuído às maiores demandas aeróbicas da modalidade esportiva.

\section{REFERÊNCIAS BIBLIOGRÁFICAS}

1. Fletcher GF, Balady GJ, Amsterdam EA, Chaitman B, Eckel R, Fleg J, et al. Exercise standards for testing and training: a statement for healthcare professionals from the American Heart Association. Circulation 2001;104(14):1694-740.

2. Meyer T, Gabriel HH, Kindermann W. Is determination of exercise intensities as percentages of VO2max or HRmax adequate? Med Sci Sports Exerc 1999;31(3):1342-5.

3. Gaskill SE, Walker AJ, Serfass RA, Bouchard C, Gagnon J, Rao DC, et al. Changes in ventilatory threshold with exercise training in a sedentary population: the Heritage family study. Int J Sports Med 2001;23(8):25-63.

4. Roecker K, Schotte O, Niess AM, Horstmann T, Dickhuth HH. Predicting competition performance in long-distance running by means of a treadmill test. Med Sci Sports Exerc 1998;30(10):1552-7.

5. Helgerud J. Maximal oxygen uptake, anaerobic threshold and running performance in women and men with similar performances levels in marathons. Eur J Appl Physiol Occup Physiol 1994;68(2):155-61.

6. Wasserman K, Mcllroy MB. Detecting the threshold of anaerobic metabolism in cardiac patients during exercise. Am J Cardiol 1964;14:844-52.

7. Meyer T, Lucia A, Earnest CP, Kinderman W. A conceptual framework for performance diagnosis and training prescription from sub maximal parameters theory and application. Int J Sports Med 2005;26(Supl 1):1-11.

8. Amann M, Subudhi J, Walker J, Eisenman P, Shultz B, Foster C. An evaluation of predictive validity and reliability of ventilatory threshold. Med Sci Sports Exerc 2004;36 (10):1716-22.

9. Hoff J, Wisløff U, Engen LC, Kemi OJ, Helgerud J. Soccer specific aerobic endurance training. Br J Sports Med 2002;36(3):218-21.

10. Casajús JA. Seasonal variation in fitness variables in professional soccer players. J Sports Med Phys Fitness 2001;41(4):463-9.

11. Al-Hazzaa HM, Almuzaini KS, Al-Refaee SA, Sulaiman MA, Dafterdar MY, Al-Ghamedi A, et al. Aerobic and anaerobic power characteristics of Saudi elite soccer players. J Sports Med Phys Fitness 2001;41(1):54-61. 
12. Díaz FJ, Montaño JG, Melchor MT, García MR Guerrero JH, Rivera AE, et al. Changes of physical and functional characteristics in soccer players. Rev Invest Clin 2003;55(5):528-34.

13. Arnason A, Sigurdsson SB, Gudmundsson A, Holme I, Engebretsen L, Bahr R. Physical fitness, injuries, and team performance in soccer. Med Sci Sports Exerc 2004;36(2):278-85.

14. Aziz AR, Mukherjee S, Chia MY, Teh KC. Relationship between measured maximal oxygen uptake and aerobic endurance performance with running repeated sprint ability in young elite soccer players. J Sports Med Phys Fitness 2007;47(4):401-7.

15. Gil S, Ruiz F, Irazusta A, Gil J, Irazusta J. Selection of young soccer players in terms of anthropometric and physiological factors. J Sports Med Phys Fitness 2007;47(1):25-32.

16. Gil SM, Gil J, Ruiz F, Irazusta A, Irazusta J. Physiological and anthropometric characteristics of young soccer players according to their playing position: relevance for the selection process. J Strength Cond Res 2007b;21(2):438-45.

17. Bunc V, Psotta R. Physiological profile of very young soccer players. J Sports Med Phys Fitness 2001;41(3):337-41.

18. Tomlin DL, Wenger HA. The relationships between aerobic fitness, power maintenance and oxygen consumption during intense intermittent exercise. J Sci Med Sport 2002;5(3):194-203.

19. Lima AMJ, Silva DVG, Souza AOS. Correlação entre as medidas direta e indireta do VO2max em atletas de futsal. Rev Bras Med Esporte 2005;11(3):164-6.

20. Leal Junior EC, Souza FB, Magini M, Lopes Martins RAB. Estudo comparativo do consumo de oxigênio e limiar anaeróbio em um teste de esforço progressivo entre atletas profissionais de futebol e futsal. Rev Bras Med Esporte 2006;12(6):323-6.
21. Castagna C, D'Ottavio S, Vera JG, Alvarez JC. Match demands of professional Futsal: A case study. J Sci Med Sport 2009;12(4):490-4.

22. McLellan TM. Ventilatory and plasma lactate response with different exercise protocols: a comparison of methods. Int J Sports Med 1985;6(1):30-5.

23. Stølen T, Chamari K, Castagna C, Wisløff U. Physiology of soccer: an update. Sports Med 2005;35(6):501-36.

24. Bangsbo J. The physiology of soccer--with special reference to intense intermittent exercise. Acta Physiol Scand Suppl 1994;619:1-155.

25. Bangsbo J. Energy demands in competitive soccer. J Sports Sci 1994b;12(special no):S5-12.

26. Bangsbo J, Mohr M, Krustrup P. Physical and metabolic demands of training and match-play in the elite football player. J Sports Sci 2006;24(7):665-74.

27. Barbero-Alvarez JC, Soto VM, Barbero-Alvarez V, Granda-Vera J. Match analysis and heart rate of futsal players during competition. J Sports Sci 2008;26(1):63-73. 